\title{
Espectro polínico da própolis de Scaptotrigona aff. postica (Hymenoptera, Apidae, Meliponini) em Barra do Corda, MA, Brasil
}

\author{
Helder Rocha de SOUZA ${ }^{1}$, Angela Maria da Silva CORRÊA², Maria Amélia Vitorino da CRUZ-BARROS², \\ Patrícia Maia Correia de ALBUQUERQUE ${ }^{*}$ \\ 1 Universidade Federal do Maranhão, Programa de Pós-Graduação em Biodiversidade e Conservação, Av. dos Portugueses, 1966, Bacanga, CEP 65080-805, São Luís, MA, Brasil. \\ 2 Instituto de Botânica, Secretaria do Meio Ambiente de São Paulo, Núcleo de Pesquisa em Palinologia, Av. Miguel Stéfano, 3687, Água Funda, CEP 04301-902, São Paulo, SP, Brasil. \\ ${ }^{3}$ Universidade Federal do Maranhão, Departamento de Biologia, Laboratório de Estudos sobre Abelhas, LEA, Av. dos Portugueses, 1966, Bacanga, CEP 65080-805, São Luis, MA, Brasil. \\ * Autor correspondente: patemaia@gmail.com
}

\section{RESUMO}

A própolis é uma substância resinosa coletada por abelhas de diferentes partes de uma planta, portanto, sua qualidade é relacionada à sua origem botânica. O objetivo deste trabalho foi verificar se os tipos polínicos encontrados indicam a origem botânica de suas resinas e contribuir com o conhecimento da relação destas abelhas com a vegetação do entorno do meliponário. Foram realizadas 31 coletas ao longo de 12 meses, com intervalo de sete dias entre elas. Os gráos de pólen foram extraídos da própolis e utilizados para confecçâo de lâminas para microscopia, posteriormente procedeu-se a identificação, contabilização e determinação das classes de frequência. Foram encontrados 94 tipos polínicos oriundos de 35 famílias botânicas. Borreria verticillata (34,17\%) foi o tipo polínico mais frequente, seguido por Anadenanthera sp. (13,65\%) e Mimosa caesalpiniifolia $(10,5 \%)$. Fabaceae $(38,37 \%)$ e Rubiaceae $(34,18 \%)$ foram as famílias que apresentaram as maiores frequências polínicas. Foram encontrados também 34 tipos polínicos exclusivos, ou seja tipos ocorrentes somente em um determinado mês do ano, podendo ser indicadores da caracterizaçáo sazonal de floração das espécies e assim inferir dados sobre sua fenologia. Os resultados obtidos náo possibilitaram a determinação da origem botânica das resinas, entretanto a grande variedade de tipos polínicos encontrados nas amostras de própolis indicou uma ampla interação entre as abelhas e as plantas e contribuíram para a caracterização fitogeográfica da própolis.

PALAVRAS-CHAVE: abelha sem ferrão, meliponicultura, pólen.

\section{Pollen spectrum of Scaptotrigona aff. postica (Hymenoptera, Apidae, Meliponini) propolis from Barra do Corda, MA, Brazil}

\begin{abstract}
Propolis is a resinous substance collected by bees from different parts of a plant. Therefore, its quality is related to its botanical origin. The objective of the study was to verify whether the pollen types found indicate the botanical origin of their resins and contribute to understand the relationship of these bees with the vegetation surrounding the meliponary. Thirty one samples were collected over 12 months at seven day intervals. Pollen grains were extracted from the propolis and used to prepare microscope slides, after which identification, counting and determination of frequency classes was undertaken. A total of 94 pollen types were identified belonging to 35 plant families. Borreria verticillata $(34.17 \%)$ was the most frequent pollen type, followed by Anadenanthera sp. (13.65\%) and Mimosa caesalpiniifolia (10.5\%). Fabaceae (38.37\%) and Rubiaceae (34.18\%) were the families with the highest pollen frequencies. There were also found 34 unique pollen types, i.e. types that only occur on a given month of the year which may be characterised as seasonal indicators for flowering species and thus infer data on their phenology. The results obtained were not enough to determine the botanical origin of the resins; however the wide variety of pollen types found in the propolis samples indicated a broad interaction between bees and plants and contributed toward the phytogeographic characterisation of the propolis.
\end{abstract}

KEYWORDS: stingless bees, beekeeping, pollen. 


\section{INTRODUÇÃO}

Estudos têm sido conduzidos na tentativa de se identificar efetivamente a origem da própolis por meio de diferentes técnicas, sejam elas diretas pela observaçáo de coleta em campo ou indiretas, por meio da análise dos constituintes químicos das estruturas anatômicas vegetais ou de análises palinológicas (Barth e Luz 2003; Barros et al. 2013; Matos et al. 2014). Esses estudos se tornaram uma ferramenta importante para a definição da qualidade e origem fitogeográfica das resinas (Barth e Luz 2003).

Grãos de pólen são encontrados na própolis, podendo ser introduzidos por abelhas operárias durante sua produçáo ou por contaminação a partir de plantas anemófilas, que se espalham com o vento, podendo ser depositados em exsudatos vegetais antes de serem coletados e levados para as colônias (Barth 2004, 2006).

Barth e Luz (2003) investigaram o espectro polínico em própolis de abelhas sem ferrão, obtidos a partir de colônias instaladas em meliponários no estado de Espírito Santo, Minas Gerais e São Paulo. Os resultados obtidos por essas autoras, mostraram que dos 60 tipos polínicos encontrados nas amostras, 22 apresentaram uma frequência maior que 3\%; e que as geoprópolis de cada um dos três estados apresentaram um ou mais tipos de pólen exclusivos. Todos estes tipos polínicos pertenciam a plantas entomófilas, o que significa que as abelhas haviam visitado estas plantas.
As análises realizadas por Teixeira et al. (2003), Bankova (2005) e Gómez-Caravaca et al. (2006), demonstraram que constituintes químicos presentes na própolis e geoprópolis podem variar em qualidade e quantidade em função da origem botânica de suas resinas, de acordo com a fitogeografia e o clima da regiáo. O conhecimento das plantas que fornecem recursos tróficos, tais como, pólen utilizado na alimentação das colônias, pode contribuir para a preservação das abelhas em ambientes naturais, agrícolas ou urbanizados (Carvalho e Marchini 1999).

O objetivo desse trabalho foi verificar se os tipos polínicos encontrados na própolis de Scaptotrigona aff. postica indicam a origem botânica de suas resinas e contribuir com o conhecimento da relação dessas abelhas com a vegetação do entorno do meliponário.

\section{MATERIAL E MÉTODOS}

\section{Local do estudo}

O presente estudo foi realizado no município de Barra do Corda, regiáo central do estado do Maranhão (530 'S, $45^{\circ} 14^{\circ} \mathrm{O}$ ), Brasil, com altitude de $81 \mathrm{~m}$, em um meliponário situado a $100 \mathrm{~m}$ da margem direita do Rio Mearim (Figura 1). Foram selecionadas e numeradas quatro colônias

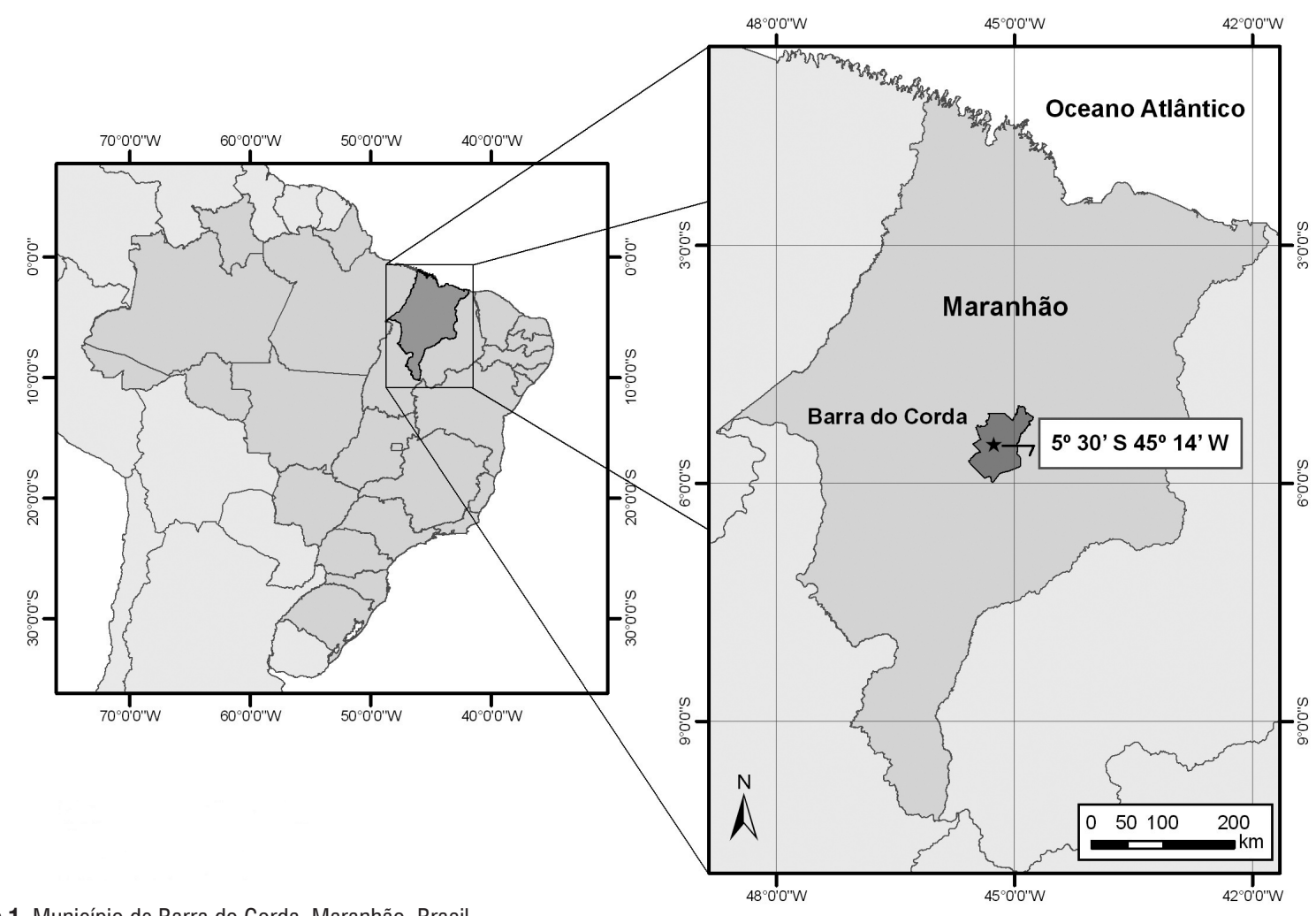

Figura 1. Município de Barra do Corda, Maranhão, Brasil. 
de Scaptotrigona aff. postica (Latreille, 1807) (Apidae, Meliponini) produtoras de própolis, com grande quantidade de crias fechadas e alimentos estocados, instaladas há mais de três anos no local; todas obtidas a partir de divisóes das colônias do próprio meliponário e instaladas em colmeias modelo "cabocla".

A tipologia climática no município é classificada como $\mathrm{C}_{1} \mathrm{SA}^{\prime} \mathrm{a}^{\prime}$ - seco subúmido, com excesso moderado no inverno e megatérmico (Maranhão 2002). No mês de maio, inicia-se a diminuiçáo das chuvas, apresentando deficiência entre junho a dezembro, com início da reposição em janeiro, apresentando excedentes entre os meses de fevereiro a abril.

A composição vegetal atual é formada por remanescentes da vegetação original em estádios secundários de sucessão e por muitas espécies que gradualmente foram introduzidas. $\mathrm{O}$ entorno do meliponário é ocupado por residências e é comum encontrar espécies de plantas ornamentais e medicinais nos quintais e nos jardins. A vegetação é constituída por um mosaico formado por pastagens, florestas abertas e vegetação degradada pela ocupação humana (Maranhão 2002). Em um raio de aproximadamente $1000 \mathrm{~m}$ do entorno do meliponário ocorre uma estreita faixa de mata ciliar nas duas margens do Rio Mearim. Uma área de aproximadamente um hectare é utilizada para o cultivo doméstico de plantas frutíferas.

\section{Coleta das amostras própolis}

As coletas das amostras de própolis de $S$. aff. postica ocorreram a cada sete dias em cada uma das quatro colônias, por um período de doze meses consecutivos, entre abril de 2011 e março de 2012, exceto no período de entressafra - início de agosto a final de novembro - totalizando 31 coletas, sempre no início da noite para se evitar o ataque das abelhas devido ao alto instinto de defesa dessa espécie. As amostras de própolis foram acondicionadas em embalagens plásticas atóxicas, devidamente identificadas com o número da colônia e data da coleta, congeladas e posteriormente enviadas ao Laboratório de Estudos Sobre Abelhas - LEA, na Universidade Federal do Maranhão - UFMA, onde permaneceram congeladas.

\section{Preparação das lâminas para análise do espectro polínico}

As amostras de própolis, após serem descongeladas, foram submetidas ao tratamento de extração do pólen utilizando-se a metodologia proposta por Barth (1998), a fim de se obter lâminas de microscopia com o material polinífero. Para elaboração das lâminas para identificação e contagem dos tipos polínicos, foi composta uma amostra com 0,125 g de própolis - coletadas no mesmo dia - de cada uma das quatro colônias. Em seguida, o material polinífero foi submetido à mistura de acetólise de Erdtman (1960).

\section{Identificação dos tipos polínicos, contagem e análise da frequência}

A identificação e contagem dos tipos polínicos foram realizadas no laboratório do Núcleo de Pesquisa em Palinologia do Instituto de Botânica de São Paulo. Foram contados no máximo 500 grãos de pólen e quando não foi possível contou-se no mínimo 300 grãos. Os tipos polínicos foram identificados por comparação, utilizando-se a Palinoteca do Núcleo de Pesquisa em Palinologia do Instituto de Botânica de São Paulo, além de trabalhos pertinentes, catálogos polínicos, e em especial para as espécies de Mimosa utilizou-se o artigo de Buril et al. (2010). Simultaneamente procurou-se estabelecer os táxons botânicos e agrupá-los por família, gênero e mais raramente por espécie.

Os grãos de pólen foram agrupados por suas similaridades morfológicas em categorias denominadas tipos polínicos caracterizados conforme o conceito de Joosten e De Klerk (2002) e De Klerk e Joosten (2007). Utilizou-se a classificação de Louveaux et al. (1978) para determinar as classes de frequências.

As fotomicrografias digitais em microscopia óptica (MO) foram realizadas utilizando-se um microscópio óptico (Olympus BX50, com câmera de vídeo Olympus SC30, Japão) acoplada a um microcomputador, utilizando-se o software CellSens Standard 1.5 de análise de imagens.

\section{RESULTADOS}

Nas análises microscópicas das lâminas com material polinífero obtidas a partir das amostras de própolis de $S$. aff. postica foi encontrada uma grande quantidade de grãos de pólen, totalizando 94 tipos polínicos distribuídos em 35 famílias (Tabela 1). Borreria verticillata (L.) G. Mey. foi o tipo polínico mais frequente (34,17\%), seguido por Anadenanthera sp. (13,65\%), Mimosa caesalpiniifolia Benth. (10,5\%), Mimosa sp.1 e Anacardium sp. (5,83\%). Fabaceae (38,37\%), Rubiaceae $(34,18 \%)$ e Anacardiaceae (7,7\%) foram as famílias que apresentaram as maiores frequências polínicas (Tabela 1, Figura 3 e Figura 4).

$\mathrm{Na}$ classe de frequência dos tipos polínicos ocasionais (abaixo de 3\%), foram encontrados 88 tipos polínicos que representaram $22,13 \%$ da quantidade de grãos de pólen contados (Tabela 1). Levando-se em consideraçáo o total de grãos de pólen contados, verificou-se que na classe de frequência dos tipos polínicos isolados (entre 3 e 15\%) foram encontrados seis tipos polínicos representando 43,7\% do total de gráos. Enquanto que na classe de frequência dos tipos polínicos acessórios (entre 15 e 45\%), foi encontrado somente um tipo polínico (Borreria verticillata) representando $34,17 \%$ do total de grãos contados (Tabela 1 ). No presente estudo náo foram encontrados tipos polínicos dominantes (acima de $45 \%$ dos grãos contados). 
Tabela 1. Frequência dos tipos polínicos encontrados em 31 amostras de própolis - Barra do Corda, MA, Brasil. Ocasional:(<3\%); Isolado:(3 - 15\%); Acessório:(16 a 45\%). Tipos polínicos exclusivos* e comuns em todos os meses**

\begin{tabular}{|c|c|c|c|c|c|c|c|c|c|c|c|}
\hline \multirow{2}{*}{ Famílias } & \multirow{2}{*}{ Tipos Polínicos } & & & & Jul & Nov & Dez & Jan & Fev & & \multirow{2}{*}{$\begin{array}{c}\% \text { total por } \\
\text { Família }\end{array}$} \\
\hline & & $\%$ & $\%$ & $\%$ & $\%$ & $\%$ & $\%$ & $\%$ & $\%$ & $\%$ & \\
\hline \multirow[t]{2}{*}{ Amaranthaceae } & & & & & & & & & & & 1,04 \\
\hline & Amaranthaceae** & 1,94 & 1,18 & 1,54 & 1,03 & 0,40 & 0,46 & 1,17 & 0,53 & 1,16 & \\
\hline \multirow[t]{6}{*}{ Anacardiaceae } & & & & & & & & & & & 7,70 \\
\hline & Anacardium ${ }^{\star *}$ & 3,65 & 3,66 & 3,29 & 3,54 & 5,35 & 10,09 & 8,15 & 9,27 & 9,60 & \\
\hline & Astronium & 0,78 & 0,49 & 0,34 & 0,96 & 2,05 & 0,20 & 0,46 & & 0,08 & \\
\hline & Mangifera & 0,39 & 2,64 & 2,86 & 1,51 & 0,15 & 0,10 & & & & \\
\hline & Schinus & & & & & 0,30 & 0,25 & & & & \\
\hline & Spondias & 0,08 & & 0,21 & 0,07 & 0,15 & & 0,07 & & 0,08 & \\
\hline \multirow[t]{2}{*}{ Apiaceae } & & & & & & & & & & & 0,06 \\
\hline & Centella & 0,23 & & & 0,07 & 0,25 & & & & & \\
\hline \multirow[t]{4}{*}{ Apocynaceae } & & & & & & & & & & & 0,03 \\
\hline & Aspidosperma* & & & & & & & 0,07 & & & \\
\hline & Mandevilla* & & & & & & 0,10 & & & & \\
\hline & Rauvolfia* & & & 0,09 & & & & & & & \\
\hline \multirow[t]{2}{*}{ Aquifoliaceae } & & & & & & & & & & & 0,01 \\
\hline & Ilex * & & 0,04 & & & & & & & & \\
\hline \multirow[t]{4}{*}{ Arecaceae } & & & & & & & & & & & 3,81 \\
\hline & Bactris $^{\star *}$ & 2,02 & 0,73 & 0,73 & 0,92 & 0,60 & 0,61 & 2,30 & 0,99 & 1,01 & \\
\hline & Arecaceae** & 0,78 & 1,83 & 0,98 & 1,88 & 0,80 & 1,22 & 0,98 & 1,52 & 0,54 & \\
\hline & Arecaceae $2^{\star \star}$ & 0,85 & 1,59 & 1,28 & 4,17 & 1,05 & 0,66 & 0,33 & 3,12 & 0,31 & \\
\hline \multirow[t]{3}{*}{ Asteraceae } & & & & & & & & & & & 0,07 \\
\hline & Vernonia & 0,16 & & & & 0,05 & & & & & \\
\hline & Asteraceae & & 0,04 & & 0,22 & & & 0,07 & & & \\
\hline \multirow[t]{2}{*}{ Bignoniaceae } & & & & & & & & & & & 0,19 \\
\hline & Tabebuia & & & 0,60 & 0,04 & 0,35 & 0,25 & 0,26 & 0,08 & & \\
\hline \multirow[t]{2}{*}{ Boraginaceae } & & & & & & & & & & & 0,01 \\
\hline & Tournefortia* & & & & & & & & 0,15 & & \\
\hline \multirow[t]{3}{*}{ Burseraceae } & & & & & & & & & & & 0,03 \\
\hline & Protium & & & 0,04 & & 0,10 & 0,05 & & & & \\
\hline & Tetragastris* & & & & & & & & & 0,08 & \\
\hline \multirow[t]{3}{*}{ Cactaceae } & & & & & & & & & & & 0,09 \\
\hline & Melocactus* & & & 0,04 & & & & & & & \\
\hline & Opuntia & & & 0,04 & 0,18 & 0,05 & 0,20 & 0,07 & 0,08 & 0,15 & \\
\hline \multirow[t]{2}{*}{ Caryophyllaceae } & & & & & & & & & & & 0,01 \\
\hline & Caryophyllaceae * & & 0,04 & & & & & & & & \\
\hline \multirow[t]{2}{*}{ Commelinaceae } & & & & & & & & & & & 0,02 \\
\hline & Commelina & & & 0,04 & 0,04 & & 0,05 & & & & \\
\hline \multirow[t]{3}{*}{ Convolvulaceae } & & & & & & & & & & & 0,63 \\
\hline & Ipomoea** & 0,62 & 1,34 & 0,68 & 0,63 & 0,15 & 0,41 & 0,52 & 0,38 & 0,62 & \\
\hline & Operculina* & & 0,04 & & & & & & & & \\
\hline \multirow[t]{2}{*}{ Cucurbitaceae } & & & & & & & & & & & 0,10 \\
\hline & Cayaponia & 0,08 & 0,12 & & 0,07 & 0,10 & 0,05 & 0,39 & 0,15 & & \\
\hline \multirow[t]{3}{*}{ Cyperaceae } & & & & & & & & & & & 0,02 \\
\hline & Cyperus* & & & 0,13 & & & & & & & \\
\hline & Cyperaceae* & & & & 0,04 & & & & & & \\
\hline
\end{tabular}


Tabela 1. Continuação

\begin{tabular}{|c|c|c|c|c|c|c|c|c|c|c|c|}
\hline \multirow{2}{*}{ Famílias } & \multirow{2}{*}{ Tipos Polínicos } & $A b r$ & Mai & Jun & Jul & Nov & Dez & Jan & Fev & Mar & \multirow{2}{*}{$\begin{array}{l}\% \text { total por } \\
\text { Família }\end{array}$} \\
\hline & & $\%$ & $\%$ & $\%$ & $\%$ & $\%$ & $\%$ & $\%$ & $\%$ & $\%$ & \\
\hline \multirow[t]{8}{*}{ Euphorbiaceae } & & & & & & & & & & & 0,86 \\
\hline & Actinostemon & & & & & 0,10 & 0,05 & & & & \\
\hline & Chamaesyce* & & 0,04 & & & & & & & & \\
\hline & Croton** & 0,54 & 0,65 & 0,64 & 0,48 & 0,45 & 0,41 & 0,78 & 0,61 & 0,54 & \\
\hline & Croton 2* & & & & & & & & 0,23 & & \\
\hline & Dalechampia & 0,08 & 0,04 & & & & & & & & \\
\hline & Mabea & & & & 0,04 & & 0,31 & 0,65 & 1,14 & 0,31 & \\
\hline & Phyllanthus & & & & & 0,05 & 0,20 & & & & \\
\hline \multirow[t]{31}{*}{ Fabaceae } & & & & & & & & & & & 38,37 \\
\hline & Acacia** & 2,41 & 1,83 & 1,45 & 1,77 & 0,85 & 0,71 & 0,26 & 2,96 & 2,48 & \\
\hline & Anadenanthera** & 10,87 & 13,34 & 8,76 & 10,47 & 13,15 & 18,59 & 18,26 & 16,41 & 17,51 & \\
\hline & Bauhinia & & & & 0,04 & & & & 7,75 & & \\
\hline & Caesalpinia & 3,73 & 3,78 & 1,71 & 0,04 & 0,10 & 0,15 & & & 0,23 & \\
\hline & Cajanus & & 0,04 & 0,04 & 0,04 & & & & & & \\
\hline & Cassia * & & & & 0,22 & & & & & & \\
\hline & Centrosema & & & 0,13 & & & 0,05 & 0,07 & & & \\
\hline & Chamaecrista & & 0,04 & & 0,07 & 0,15 & 0,25 & & & & \\
\hline & Copaifera & 0,54 & 0,12 & 0,30 & 0,74 & 0,30 & 0,87 & 0,13 & & & \\
\hline & Dalbergia & & 0,04 & & & 0,05 & 0,15 & 0,59 & 0,08 & & \\
\hline & Delonix * & & & & & & & & & 0,08 & \\
\hline & Desmodium & 0,16 & 0,28 & 0,04 & 0,07 & 0,05 & & & 0,08 & & \\
\hline & Entada ** & 2,48 & 2,56 & 1,71 & 0,66 & 1,05 & 0,51 & 1,70 & 1,29 & 1,16 & \\
\hline & Galactia & & & & 0,04 & & & 0,07 & 0,08 & & \\
\hline & Hymenaea & & 0,08 & & 0,07 & & & & & & \\
\hline & Inga & & & & & & 0,05 & & & 0,15 & \\
\hline & Leucaena * & & & & & 0,05 & & & & & \\
\hline & Machaerium & 0,16 & 1,02 & 0,43 & 0,18 & 0,70 & & & & 0,15 & \\
\hline & $\begin{array}{c}\text { Mimosa } \\
\text { caesalpiniifolia** }\end{array}$ & 14,36 & 14,80 & 17,00 & 8,19 & 7,05 & 6,72 & 8,48 & 7,83 & 7,67 & \\
\hline & Mimosa invisa ${ }^{\star \star}$ & 1,40 & 0,61 & 1,79 & 0,85 & 1,15 & 0,56 & 0,78 & 0,53 & 1,01 & \\
\hline & Mimosa $1^{* *}$ & 9,01 & 8,62 & 8,63 & 2,54 & 4,75 & 8,46 & 4,62 & 8,05 & 5,89 & \\
\hline & Mucuna * & & & & & 0,05 & & & & & \\
\hline & Phaseolus & 0,16 & 0,08 & & & & & & & & \\
\hline & Plathymenia * & & & & & & & & 0,08 & & \\
\hline & Senna & & & 0,04 & 0,04 & & 0,05 & 0,26 & & & \\
\hline & Stizolobium * & & & & & 0,05 & & & & & \\
\hline & Stylosanthes * & & & & & & & 0,07 & & & \\
\hline & Swartzia * & & & & 0,04 & & & & & & \\
\hline & Vatairea * & & & & & 0,10 & & & & & \\
\hline & Fabaceae & 0,39 & & 0,21 & 0,55 & 0,55 & 1,27 & 0,98 & 1,37 & 2,32 & \\
\hline \multirow[t]{2}{*}{ Flacourtiaceae } & & & & & & & & & & & 0,01 \\
\hline & Flacourtiaceae* & & & & & 0,05 & & & & & \\
\hline \multirow[t]{2}{*}{ Lamiaceae } & & & & & & & & & & & 0,23 \\
\hline & Hyptis & & 0,08 & 0,43 & 0,63 & 0,05 & 0,05 & & 0,08 & 0,54 & \\
\hline \multirow[t]{2}{*}{ Lythraceae } & & & & & & & & & & & 0,11 \\
\hline & Lythraceae* & & & & & & 0,92 & & & & \\
\hline
\end{tabular}


Tabela 1. Continuação

\begin{tabular}{|c|c|c|c|c|c|c|c|c|c|c|c|}
\hline Famílias & Tipos Polínicos & $\begin{array}{c}\text { Abr } \\
\%\end{array}$ & $\begin{array}{c}\text { Mai } \\
\%\end{array}$ & $\begin{array}{l}\text { Jun } \\
\%\end{array}$ & $\begin{array}{l}\text { Jul } \\
\%\end{array}$ & $\begin{array}{l}\text { Nov } \\
\%\end{array}$ & $\begin{array}{c}\text { Dez } \\
\%\end{array}$ & $\begin{array}{l}\text { Jan } \\
\%\end{array}$ & $\begin{array}{c}\text { Fev } \\
\%\end{array}$ & $\begin{array}{l}\text { Mar } \\
\%\end{array}$ & $\begin{array}{l}\% \text { total por } \\
\text { Família }\end{array}$ \\
\hline \multirow[t]{3}{*}{ Malpighiaceae } & & & & & & & & & & & 0,04 \\
\hline & Stigmaphyllon & & 0,20 & 0,04 & & & & & & & \\
\hline & Malpighiaceae* & & & & & & & 0,07 & & & \\
\hline \multirow[t]{3}{*}{ Malvaceae } & & & & & & & & & & & 0,01 \\
\hline & Helicteres & & & & 0,04 & & & & & & \\
\hline & Pachira & & & & 0,04 & & & & & & \\
\hline \multirow[t]{2}{*}{ Melastomataceae } & & & & & & & & & & & 3,99 \\
\hline & Melastomataceae** & 5,28 & 5,49 & 2,95 & 1,99 & 2,85 & 4,43 & 3,32 & 6,08 & 5,73 & \\
\hline \multirow[t]{3}{*}{ Meliaceae } & & & & & & & & & & & 0,04 \\
\hline & Cedrela & & & & 0,04 & & & 0,07 & & & \\
\hline & Trichilia & & & & 0,07 & & & 0,13 & & & \\
\hline \multirow[t]{2}{*}{ Myrtaceae } & & & & & & & & & & & 3,15 \\
\hline & Myrtaceae** & 2,87 & 2,36 & 2,73 & 1,40 & 4,80 & 3,92 & 2,66 & 4,86 & 4,49 & \\
\hline \multirow[t]{2}{*}{ Nyctaginaceae } & & & & & & & & & & & 0,01 \\
\hline & Boerhavia * & & & & & 0,05 & & & & & \\
\hline \multirow[t]{2}{*}{ Phyllanthaceae } & & & & & & & & & & & 0,01 \\
\hline & Hyeronima * & & & & & & 0,05 & & & & \\
\hline \multirow[t]{2}{*}{ Poaceae } & & & & & & & & & & & 0,07 \\
\hline & Poaceae & & & 0,26 & 0,11 & & 0,10 & & & & \\
\hline \multirow[t]{4}{*}{ Rubiaceae } & & & & & & & & & & & 34,18 \\
\hline & Borreria verticillata** & 31,91 & 27,56 & 34,94 & 47,16 & 42,95 & 30,06 & 33,82 & 17,25 & 29,28 & \\
\hline & Psychotria & & & & & 0,05 & & & & & \\
\hline & Rubiaceae* & & & & & & 0,05 & & & & \\
\hline \multirow[t]{3}{*}{ Rutaceae } & & & & & & & & & & & 1,78 \\
\hline & Citrus & 0,39 & 0,37 & 0,38 & 0,55 & 0,20 & 0,36 & & 0,46 & 0,54 & \\
\hline & Zanthoxylum & & 0,04 & 0,13 & 0,81 & 2,30 & 2,55 & 3,32 & 2,74 & 2,32 & \\
\hline \multirow[t]{2}{*}{ Salicaceae } & & & & & & & & & & & 0,01 \\
\hline & Casearia * & & & & & 0,05 & & & & & \\
\hline \multirow[t]{7}{*}{ Sapindaceae } & & & & & & & & & & & 1,92 \\
\hline & Cupania * & & & & & 0,20 & & & & & \\
\hline & Matayba * & & & & & 0,05 & & & & & \\
\hline & Paullinia & & & 0,17 & & 0,25 & & & & & \\
\hline & Talisia & & & & & 0,30 & 0,20 & & 0,08 & & \\
\hline & Serjania ** & 1,09 & 0,85 & 0,77 & 2,58 & 1,75 & 1,12 & 2,02 & 1,44 & 1,24 & \\
\hline & Sapindaceae Indet & & 0,12 & 0,09 & 0,59 & 0,15 & 0,36 & 0,52 & 0,30 & 0,85 & \\
\hline \multirow[t]{2}{*}{ Sapotaceae } & & & & & & & & & & & 0,01 \\
\hline & Pouteria & 0,08 & & 0,04 & & & & & & & \\
\hline \multirow[t]{2}{*}{ Solanaceae } & & & & & & & & & & & 0,52 \\
\hline & Solanaceae** & 0,08 & 1,02 & 0,64 & 0,77 & 0,45 & 0,46 & 0,07 & 0,46 & 0,08 & \\
\hline \multirow[t]{2}{*}{ Indeterminados } & & 0,47 & 0,08 & 0,64 & 0,70 & 0,85 & 1,27 & 1,43 & 1,52 & 1,78 & 0,88 \\
\hline & Total (\%) & 100 & 100 & 100 & 100 & 100 & 100 & 100 & 100 & 100 & 100 \\
\hline \multicolumn{2}{|c|}{ Total de Tipos polínicos identificados no mês } & 33 & 41 & 44 & 51 & 53 & 47 & 38 & 35 & 32 & - \\
\hline \multicolumn{2}{|c|}{ Total de Tipos polínicos exclusivos no mês } & 0 & 4 & 3 & 5 & 10 & 4 & 3 & 3 & 2 & - \\
\hline
\end{tabular}


Entre os tipos polínicos encontrados, observou-se que 34 deles ocorreram exclusivamente em um determinado mês do ano. Observa-se na Figura 2 que ocorreram dois picos de tipos polínicos exclusivos, o menor em maio e o maior em novembro. No mês de maio ocorreram quatro tipos polínicos exclusivos: Operculina sp., Caryophyllaceae, Ilex sp. e Chamaesyce sp.; em junho ocorreram três tipos polínicos exclusivos: Rauvolfia sp., Melocactus sp. e Cyperus sp.; em julho cinco tipos: Cassia sp., Cyperaceae, Helicteres sp., Pachira sp. e Swartzia sp.; em novembro dez tipos: Leucaena sp., Mucuna sp., Stizolobium sp., Vatairea sp., Flacourtiaceae, Boerhavia sp., Casearia sp., Cupania sp., Matayba sp. e Psychotria sp.; em dezembro quatro tipos: Mandevilla sp., Lythraceae, Hyeronima sp. e Rubiaceae; em janeiro três tipos: Malpighiaceae, Stylosanthes sp. e Aspidosperma sp.; em fevereiro três tipos: Tournefortia sp., Croton 2 sp. e Plathymenia sp.; e em março ocorreram dois tipos polínicos exclusivos: Tetragastris sp. e Delonix sp. Dezenove tipos polínicos ocorreram em todos os meses em que houve amostragem (Tabela 1, Figura 2, Figura 3 e Figura 4).

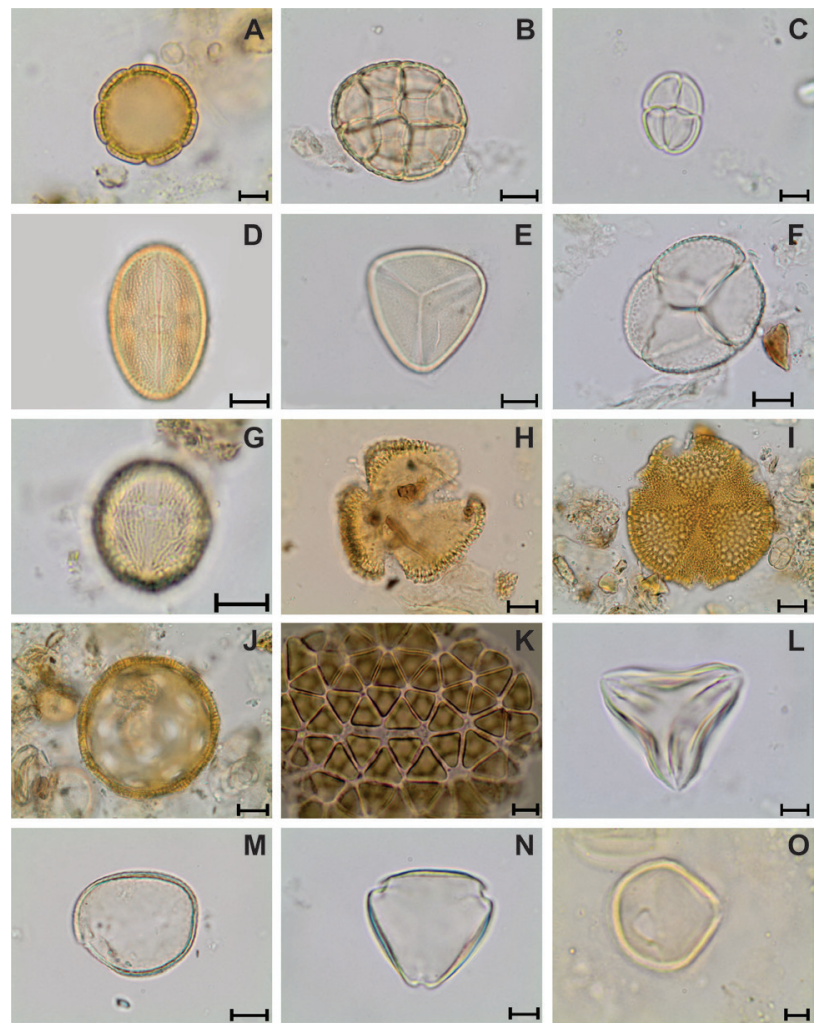

Figura 3. Alguns tipos polínicos encontrados em amostras de própolis de Scaptotrigona aff. postica, em Barra do Corda, MA, Brasil: A. Borreria verticillata B. Anadenanthera C. Mimosa caesalpiniifolia D. Anacardium E. Arecaceae F. Mimosa 1 G. Astronium H. Bignoniaceae I. Caesalpinia J. Caryophyllaceae K. Croton L. Copaifera M. Monocotiledônea N. Myrtaceae 0. Dalbergia (Escalas: B, D, E, G, H, J, K, M, $0=10 \mu \mathrm{m}$; A, C, F, I, L, N = 5 $\mu \mathrm{m})$. Esta figura é colorida na versão eletrônica.

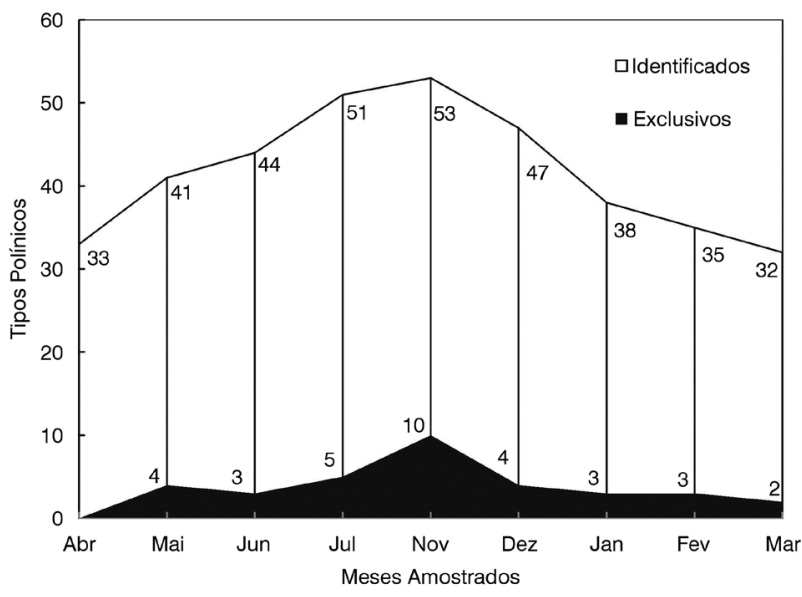

Figura 2. Quantidade de tipos polínicos identificados e tipos polínicos exclusivos, encontrados na própolis de Scaptotrigona aff. postica (Latreille, 1807) em Barra do Corda, MA, Brasil no período entre abril de 2011 e março de 2012.
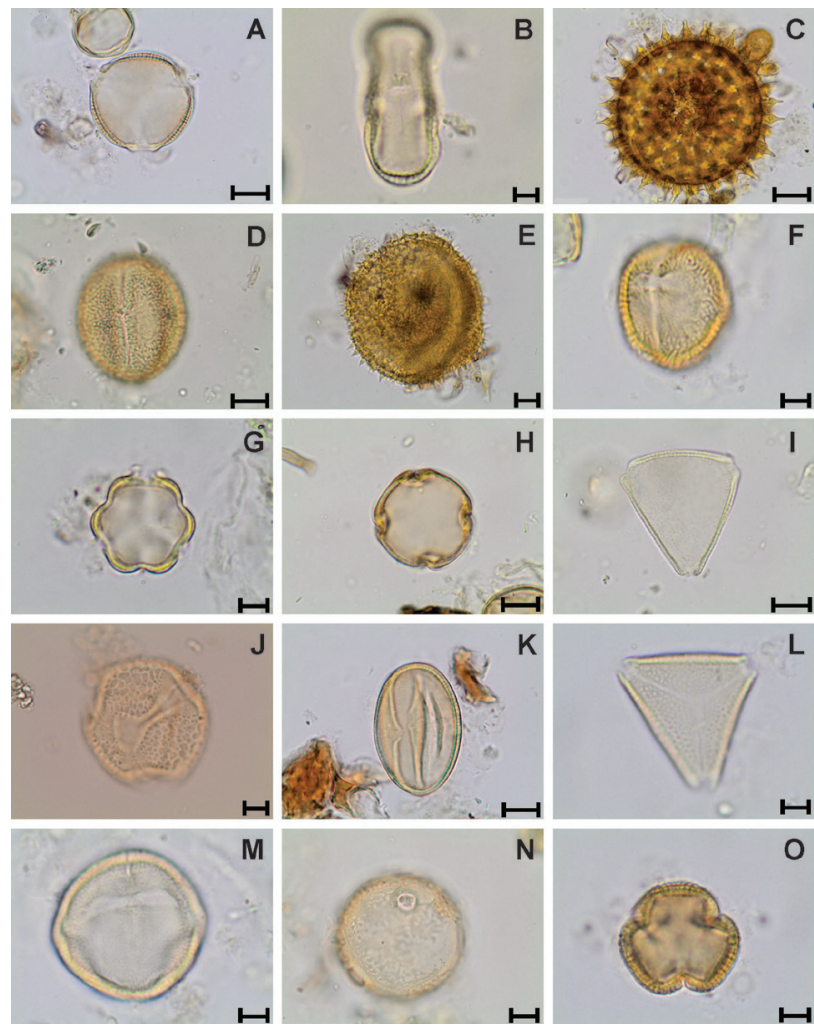

Figura 4. Alguns tipos polínicos encontrados em amostras de própolis de Scaptotrigona aff. postica, em Barra do Corda, MA, Brasil: A. Entada B. Hieronima C. Ipomoea D. Mabea E. Malvaceae F. Mangifera G. Melastomataceae H. Meliaceae I. Paullinia J. Phaseolus K. Senna L. Serjania M. Solanaceae N. Stigmaphyllon O. Zanthoxylum (Escalas: A, B, C, I = 20 $\mu \mathrm{m} ; \mathrm{D}, \mathrm{H}, \mathrm{J}, \mathrm{K}, \mathrm{N}, \mathrm{O}=10 \mu \mathrm{m} ; \mathrm{E}, \mathrm{F}, \mathrm{G}, \mathrm{L}, \mathrm{M}=5 \mu \mathrm{m})$. Esta figura é colorida na versão eletrônica. 


\section{DISCUSSÃO}

A presença de tipos polínicos, predominantemente entomófilos, na própolis de $S$. aff. postica ocorreu em função do contato das abelhas com as flores de uma grande diversidade de espécies vegetais. Isto demonstra uma importante interação entre as abelhas e as plantas no processo de polinização e utilização de recursos florais, que é um dos fatores que favorecem a manutenção de comunidades de abelhas em áreas urbanizadas (Taura e Laroca 1991). Foi detectado, também a presença em pequena quantidade, de tipos polínicos anemófilos das famílias Cyperaceae (Cyperus sp.) e Poaceae. A ocorrência desses tipos polínicos, provavelmente se deu pela contaminação da própolis da colmeia pelo pólen do ar, e cuja planta, fonte de resina, não estava realmente acessível às abelhas conforme observado por Barros et al. (2013).

Grande parte dos tipos polínicos encontrados na própolis de Scaptotrigona aff. postica foram considerados pólen ocasional. Tipos polínicos de baixa ocorrência podem significar plantas pouco produtoras de pólen ou plantas pouco atrativas para as abelhas (Barros et al. 2013). Entretanto para Freitas et al. (2010) os tipos polínicos com baixa frequência podem ser indicativos de possíveis espécies fornecedoras de resina. Independente disso, esses tipos polínicos são importantes para a caracterização fitogeográfica da região dessas plantas (Freitas et al. 2010).

Entre os tipos polínicos encontrados na própolis da Tubi (Scaptotrigona aff. postica) no Maranhão, destaca-se Borreria verticillata pertencente à família Rubiaceae, cujo gênero possui em torno de 100 espécies (Cabral 1991) distribuídas em regióes tropicais e subtropicais da América, África, Ásia e Austrália e, sua maior diversidade, encontrase no Brasil (Steyermark 1972). Em estudos sobre recursos florais utilizados por abelhas sociais Gonçalves et al. (1996), observaram que $B$. verticillata foi a espécie vegetal mais visitada pelas abelhas no município de Alcântara, estado do Maranhão. $\mathrm{Na}$ região de Barra do Corda, esta planta é considerada uma erva daninha que invade áreas de cultivo agrícola ou em quintais e jardins de residências. Suas flores são comumente visitadas por diversas espécies de abelhas nativas, e em todas as amostras de própolis foi o tipo polínico mais encontrado. Esta planta pode representar grande importância trófica para $S$. aff. postica no local onde ocorreu este estudo, dada a sua presença constante e alta frequência nas amostras de própolis coletada ao longo da amostragem. Segundo Matos et al. 2014, em estudos sobre compostos fenólicos em própolis produzida por Apis mellifera L. (Apidae), houve uma correlação positiva com os tipos identificados como Asteraceae I, Borreria verticillata, Mikania e Mitracarpus longicalyx em uma área do semiárido da região da Bahia. Entretanto os autores não concluiram que esses resultados tenham sido suficientes para afirmar que a associação da correlação dos níveis de compostos fenólicos totais com esses tipos polínicos seja realmente válido para própolis produzida na região do estudo, necessitando de outros estudos com mais amostras.

A ocorrência exclusiva de tipos polínicos em um determinado mês do ano pode ser utilizada como indicador da caracterização sazonal de floração das espécies, e assim inferir dados sobre sua fenologia, a qual segundo Andreis et al. (2005), está relacionada às condiçóes climáticas e ao caráter adaptativo de cada espécie em sua área de dispersáo.

\section{CONCLUSÕES}

Tanto as análises quantitativas quanto as qualitativas dos tipos polínicos encontrados na própolis de Scaptotrigona aff. postica foram valiosas para a sua caracterização. Os resultados obtidos fornecem subsídios para se conhecer as possíveis espécies fornecedoras de resina para a Tubi, ampliando os conhecimentos científicos sobre a própolis das abelhas sem ferrão que ainda são incipientes, podendo também contribuir para a proteção dessa espécie de abelha na elaboração de programas de conservação e recomposição vegetal de suas áreas de ocupação.

\section{AGRADECIMENTOS}

Nossos agradecimentos ao meliponicultor Wilson Melo por disponibilizar suas colônias de Scaptotrigona aff. postica, à J.P. Silva por produzir o mapa da Figura 1, à P.O.B. Souza pela revisão textual, à Fundação de Amparo à Pesquisa e Desenvolvimento Científico do Maranhão - FAPEMA, (Processo no PAEDT-01313/11), pelo apoio financeiro e à Coordenação de Aperfeiçoamento de Pessoal de Nível Superior CAPES, no âmbito do PNADB - Programa Nacional de Apoio ao Desenvolvimento da Botânica, por ter proporcionado a parceria com o Instituto Botânico de São Paulo.

\section{BIBLIOGRAFIA CITADA}

Andreis C.; Longhi, S. J.; Brun, E. J.; Wojciechowsk, J. C.; Machado, A. A.; Vaccaro, S.; Cassal, C. Z. 2005. Estudo fenológico em três fases sucessionais de uma floresta estacional decidual no município de Santa Tereza, RS, Brasil. Revista Árvore, 29:55-63.

Bankova, V. 2005. Chemical diversity of propolis and the problem of standardization. Journal of Ethnopharmacology, 100:114-117.

Barros, M.H.M.R.; Luz, C.F.P.; Albuquerque, P.M.C. 2013. Pollen analysis of geopropolis of Melipona (Melikerria) fasciculata Smith, 1854 (Meliponini, Apidae, Hymenoptera) in areas of resting, Cerrado and flooded fields in the state of Maranhão, Brazil. Grana, 52: 81-92.

Barth, O.M. 1998. Pollen analysis of Brazilian propolis. Grana, 37: $97-101$.

Barth, O.M. 2004. Melissopalynology in Brazil: a review of pollen analysis of honey, propolis and pollen loads of bees. Scientia Agricola, 61:342 - 350. 
Barth, O.M. 2006. Palynological analysis of geopropolis samples obtained from six species of Meliponinae in the Campus of the Universidade de Ribeirão Preto, USP, Brasil. Apiacta, 1: 1-14.

Barth, O.M.; Luz, C.F.P. 2003. Palynological analysis of Brazilian geoprópolis sediments. Grana, 42:121-127.

Buril, M.T.; Santos, F.A.R.; Alves, M. 2010. Diversidade polínica das Mimosoideae (Leguminosae) ocorrentes em uma área de caatinga, Pernambuco, Brasil. Acta Botanica Brasilica, 24: 53-64.

Cabral, E.L. 1991. Rehabilitación del gênero Galianthe (Rubiaceae). Boletin de la Sociedad Argentina de Botanica, 27:217-231.

Carvalho, C.A.L.; Marchini, L.C. 1999. Tipos polínicos coletados por Nannotrigona testaceicornis e Tetragonisca angustula (Hymenoptera, Apidae, Meliponinae). Scientia Agricola, 56:717-722.

De Klerk, P.; Joosten, H. 2007. The difference between pollen types and pollen taxa: a plea for clarity and scientific freedom. Quaternary Science Journal, 56: 24-33.

Erdtman, G. 1960. The acetolysis method: a revised description. Svensk Botanisk Tidskrift, 54: 561-564.

Freitas A.S.; Barth M.O.; Luz, C.F.P. 2010. Própolis marrom da vertente atlântica do Estado do Rio de Janeiro, Brasil: uma avaliação palinológica. Revista Brasileira de Botânica, 33: 343-354.

Gómez-Caravaca A.M.; Gómez-Romero M.; Arráez-Román, D.; Segura-Carretero, A.; Fernández-Gutiérrez, A. 2006. Advances in the analysis of phenolic compounds in products derived from bees. Journal of Pharmaceutical and Biomedical Analysis, 41:1220-1234.

Gonçalves, S.J.M.; Rêgo, M.; Araújo, A. 1996. Abelhas sociais (Hymenoptera: Apidae) e seus recursos florais em uma região de mata secundária, Alcântara, MA, Brasil. Acta Amazonica, 26: 55-68.
Joosten, H.; De Klerk, P. 2002. What's in a name? Some thoughts on pollen classification, identification, and nomenclature in Quaternary palynology. Review of Palaeobotany and Palynology, 122: 29-45.

Louveaux, J.; Maurizio, A.; Vorwohl, G. 1978. Methods of melissopalynology. Bee World, 59:139-157.

Maranhão. 2002. Atlas do Maranhão / Gerência de Planejamento e Desenvolvimento Econômico, Laboratório de Geoprocessamento - UEMA. São Luís: GEPLAN / UEMA. 44p.

Matos, V.R.; Alencar, S.M.; Santos, F.A.R. 2014. Pollen types and levels of total phenolic compounds in propolis produced by Apis mellifera L. (Apidae) in an area of the Semiarid Region of Bahia, Brazil. Anais da Academia Brasileira de Ciências, 86: 407-418.

Steyermark, J.A. 1972. Borreria G.F. Meyer. The botany of the Guayana highland, part IX. Memoirs of the New York Botanical Garden, 23:805-831.

Taura H.M.; Laroca S. 1991. Abelhas altamente sociais (Apidae) de uma área restrita em Curitiba (Brasil): distribuição dos ninhos e abundância relativa. Acta Biológica Paranaense, 20: 85 - 101.

Teixeira E.W.; Message D.; Meira R.M.S.A.; Salatino, A. 2003. Indicadores da origem botânica da própolis, importância e perspectivas. Revisão bibliográfica. Boletim da Indústria Animal, 60:83-106.

Recebido em 18/09/2014

Aceito em 21/01/2015 
